\title{
Quantum secret sharing based on reusable GHZ states as secure carriers
}

\author{
Saber Bagherinezhad ${ }^{a *}$ \\ Vahid Karimipour ${ }^{b \dagger}$ \\ ${ }^{a}$ Department of Computer Science, Sharif University of Technology, \\ ${ }^{b}$ Department of Physics, Sharif University of Technology \\ P. O. Box 11365-9161, Tehran, Iran
}

October 30, 2018

\begin{abstract}
We introduce a protocol for quantum secret sharing based on reusable entangled states. The entangled state between the sender and the receiver acts only as a carrier to which data bits are entangled by the sender and disentangled from it by the receivers, all by local actions of simple gates. We also show that the interception by Eve or the cheating of one of the receivers introduces a quantum bit error rate (QBER) larger than 25 percent which can be detected by comparing a subsequence of the bits.
\end{abstract}

Keywords: Secret sharing, entanglement, quantum cryptography.

\footnotetext{
*e-mail:bagherin@ce.sharif.ac.ir
}

†e-mail: vahid@sina.sharif.ac.ir. 


\section{Introduction}

The past few years has witnessed progress in theoretical aspects and experimental implementations of quantum cryptography. (For an elementary introduction to the subject see [1] and for a comprehensive review of recent advances see [2.)

One of the desirable protocols for secure communication is called secret sharing, the simplest of which is when the sender Alice wants to send a secret message to two receivers Bob and Charlie so that non of the receivers can recover the message on his own. In 1998, Hillery, Buzek, and Berthiaume proposed a quantum solution for secret sharing [3]. In their method which is inspired by the quantum key distribution method of Bennett and Brasard [4, and Ekert [5], Alice, Bob and Charlie share a GreenbergerHorne-Zeilinger (GHZ) state 6]. They then carry out measurements of their bits in either of the two non-commuting bases, say $x$ and $y$ bases in random. Since the results or measurements are correlated for half of the cases, they can establish a secret key between themselves by announcing their bases of measurements. The aim of the present article which is inspired by the work of Zhang, Li and Guo [7] in quantum key distribution, is to present an alternative method for secret sharing, which is based on sharing entangled states as carriers to which data bits are entangled by the sender Alice and disentangled by the receivers Bob and Charlie. The role of this carrier is to make communication secure against intervention of evesdroppers or cheating of any of the receivers.

Two remarks are in order comparing the differences of our protocols with the known ones [3, 8].

1- If experimental realization of constructing and distributing $G H Z$ states to parties at long separation becomes a possibility in the future, it is plausible to assume that the maintenance of the correlations of these states will be easier and less costly than their creation anew for every round as is needed in earlier protocols for secret sharing. 2- We should also point out that this protocol is not far from the reach of the near future experiments. At present single qubit gates and double qubit gates very close to the CNOT gate, can be implemented on individual ions where up to ten ions are kept in a coherent state [9]. One can also imagine that by methods similar to the ones proposed in 10], distant atoms or ions can be entangled with each other in the near future. The structure of this paper is as follows: In section 2 we introduce the protocol for two receiving parties, and discuss how information is split and is protected from unauthorized parties. We also show how intervention of Eve can be detected. We end up the paper with conclusions.

\section{The secret sharing protocol with two parties}

Suppose Alice wants to sent a message which is already in the form of a sequence of classical bits $q_{1}, q_{2}, q_{3}, \cdots,\left(q_{i}=0\right.$ or 1$)$ to Bob and Charlie, so that the receivers can infer this message only by their mutual assistance. We begin with our notations. We use subscripts $a, b, c$ and $e$ on states and operators for Alice, Bob, Charlie, and Eve respectively. Any other space carrying message qubits is specified by numerical 
subscripts, 1, 2, etc. A controlled gate like CNOT is denoted simply by $C$, and is specified by two subscripts, the first one is the control bit, the second is the target bit. Thus $C_{a 1}$ is the controlled NOT gate which is controlled by Alice qubit and acts on the qubit in line 1, i.e: $C_{a 1}\left|q, q^{\prime}\right\rangle_{a 1}=\left|q, q+q^{\prime}\right\rangle_{a 1}$, where $q$ and $q^{\prime}$ are 0 and 1 and addition is performed mod two.

The Hadamard gate acts as $H|0\rangle=\frac{1}{\sqrt{2}}(|0\rangle+|1\rangle)$ and $H|1\rangle=\frac{1}{\sqrt{2}}(|0\rangle-|1\rangle)$. By $|\overline{0}\rangle$ and $|\overline{1}\rangle$, we mean two qubit states which are uniform superposition of basis states the sum of whose digits modulo 2 are respectively 0 and 1, i.e: $|\overline{0}\rangle=\frac{1}{\sqrt{2}}(|0,0\rangle+|1,1\rangle)$ and $|\overline{1}\rangle=\frac{1}{\sqrt{2}}(|0,1\rangle+|1,0\rangle)$, which can be identified only by the collaboration of the two parties possessing the bits.

Moreover the following property is also easily verified:

$$
C_{a 1} C_{b 2}|\bar{q}\rangle_{a, b}\left|\overline{q^{\prime}}\right\rangle_{1,2}=|\bar{q}\rangle_{a, b}\left|\overline{q+q^{\prime}}\right\rangle_{1,2}
$$

We also need to define two three-particle states, namely the GHZ state which we denote by

$$
|G\rangle:=\frac{1}{\sqrt{2}}(|000\rangle+|111\rangle) .
$$

and an even parity state which we denote by

$$
|E\rangle:=\frac{1}{2}(|000\rangle+|110\rangle+|101\rangle+|011\rangle) \equiv \frac{1}{\sqrt{2}}(|0\rangle|\overline{0}\rangle+|1\rangle|\overline{1}\rangle) .
$$

These two states are transformed to each other by the local operation of Hadamard gates, that is:

$$
|G\rangle=H \otimes H \otimes H|E\rangle \quad \text { and } \quad|E\rangle=H \otimes H \otimes H|G\rangle
$$

We will use these two states which are shared by all three parties, as carriers of information, (the $|G\rangle$ state in the odd rounds and the $|E\rangle$ state in the even rounds). Alice entangles her data bits to the above carriers and bob and Charlie disentangle the data bits from these carriers. Due to property (4), the action of Hadamard gates performed by all the parties at the end of each round switches the Carrier to the appropriate one for the next round. This switching of carriers is also crucial for the security of the protocol as we will see in the sequel.

For sending a classical bit $q$ Alice may encode it as a state $|q, q\rangle$ and send this state simply to Bob and Charlie. At the destination Bob and Charlie can measure their corresponding bits and recover the bit $q$. In this case Bob and Charlie can understand $q$ without each other's assistance, and in fact Alice sends half of the bits say the odd numbered ones $q_{1}, q_{3}, q_{5} \cdots$ in this way. For the other half, $q_{2}, q_{4}, q_{6} \cdots$, she encodes a bit $q$ in the form of a state $|\bar{q}\rangle=\frac{1}{\sqrt{2}}(|0, q\rangle+|1, q+1\rangle)$ and sends it to Bob and Charlie who are assumed to have access to the first and second spaces respectively. Any such state gives no information at all to either Bob or Charlie, since the density matrix of each of them is easily seen to be completely mixed. However they can identify the bit $q$ by communicating to each other the result of their measurements. The value of the bit $q$ is simply obtained by adding their result mod 2. In this way Alice can split a 
message so that Bob and Charlie can recover the message only by their cooperation. A cheating of the kind of wrong declaration of the results by one of the receivers leads to 50 percent errors which is easily detected by comparing a subsequence of the bits received with those actually sent by Alice. By this comparison Alice finds that at least one of the receivers has been dishonest. Although she can not determine which one.

This is the part of protocol which deals with splitting of information. Now we are faced with the problem of protecting information against Eavesdropping and against cheating of one of the parties who may find access via the collaboration of Eve to both the qubits.

We should assume that the quantum channel used by Alice for sending the qubits is not secure and can be penetrated by an unauthorized third party called Eve (who incidentally may be one of the dishonest receivers say Bob) finding access to both of the bits in transition and retrieving the data (without assistance of Charlie). We now make our protocol safe against such attacks or cheating. The strategy is to entangle the message qubits with an already entangled state in possession of Alice, Bob and Charlie, in a highly mixed form, so that while being sent, these qubits if accessed by Eve or by one of the receivers say Bob, carry no information at all. Moreover we should also show that Eve's intervention and Bob's cheating can be detected by the other parties. We use two different forms of carriers for odd and even bits. For odd bits, we proceed as follows. Alice entangles the state $|q q\rangle_{12}$ to the already present GHZ state $|G\rangle_{a b c}$ by performing CNOT gates $C_{a 1} C_{a 2}$ on

$$
|G\rangle_{a b c}|q q\rangle_{12}=\frac{1}{\sqrt{2}}(|0,0,0\rangle+|1,1,1\rangle)_{a, b, c}|q, q\rangle_{12}
$$

to produce the state:

$$
\left|\Phi^{\text {odd }}\right\rangle=\frac{1}{\sqrt{2}}\left(|0,0,0\rangle_{a b c}|q, q\rangle_{12}+|1,1,1\rangle_{a, b, c}|1+q, 1+q\rangle_{12}\right)
$$

At the destination, Bob and Charlie act on this state by the operators $C_{b 1}$ and $C_{c 2}$ and extract the state $|q, q\rangle_{1,2}$ where each one of them can read independently his own bit. By her action Alice has entangled the double bit $|q, q\rangle_{1,2}$ so that while in transmission it is a mixture of $|q, q\rangle$ and $|1+q, 1+q\rangle$ which conveys no information to Eve about the value of the bit being sent.

It is also seen from the state (6) that a simple intercept-resend strategy adopted by Eve of the two flying data qubits, will make 50 percent error in the data bits jointly received by Bob and Charlie with those sent by Alice. Therefore Eve's presence can be detected by publicly comparing a subsequence of the bits sent by Alice with those received by Bob and Charlie.

For the even bits which are encoded as states $|\bar{q}\rangle$, i.e. $\left(|\overline{0}\rangle=\frac{1}{\sqrt{2}}(|00\rangle+|11\rangle)\right.$ and $\left.|\overline{1}\rangle=\frac{1}{\sqrt{2}}(|01\rangle+|10\rangle)\right)$, Alice entangles this state to the carrier $|E\rangle$ by performing only one single CNOT gate $C_{a 1}$ on

$$
|E\rangle_{a b c} \otimes|\bar{q}\rangle_{12}=\frac{1}{\sqrt{2}}(|0\rangle|\overline{0}\rangle+|1\rangle|\overline{1}\rangle)_{a b c}|\bar{q}\rangle_{1,2}
$$


to produce the state

$$
\left|\Psi^{e v e n}\right\rangle=\frac{1}{\sqrt{2}}\left(|0\rangle_{a}|\overline{0}\rangle_{b c}|\bar{q}\rangle_{12}+|1\rangle_{a}|\overline{1}\rangle_{b c}|\overline{1+q}\rangle_{12}\right)
$$

where we have used the the property (11). At the destination, Bob and Charlie act on this state by the operators $C_{b 1}$ and $C_{c 2}$ where again by (11) they extract $|\bar{q}\rangle_{1,2}$ which they can identify completely only by their collaboration. It is quite simple to see from (8) that $\rho_{b 1}=\rho_{c 2}=\rho_{12}=\frac{1}{2} I$.

This means that neither Eve alone who may supposedly find access to the two data bits nor any of the receivers independently, can find the data bit which has been encoded and sent by Alice. For the even rounds the simple intercept-resend strategy of Eve introduces 50 percent discrepancy among the data bits of Bob with those of Charlie which again leads to the detection of Eve, since a state which has been encoded as $|\overline{0}\rangle=\frac{1}{\sqrt{2}}(|00\rangle+|11\rangle)$ is received half the time by Bob and Charlie as a state $|\overline{1}\rangle=\frac{1}{\sqrt{2}}(|01\rangle+|10\rangle)$.

Note that once the data bits are measured by Bob and Charlie, only one of them needs to publicly announce the result of his measurement, and the other will find the actual bit sent by Alice, by simply adding the bit publicly announced to the one that he has actually measured. This public announcement again does not convey any information to Eve. Moreover a wrong declaration of results by one of the receivers say Bob again leads to discrepancies of the bits between Alice and Charlie.

Before going on to study a general attack of Eve or Cheating of Bob, lets finish the protocol by saying how Alice, Bob and Charlie switch their entangled state from the GHZ state $|G\rangle_{a, b, c}$ for odd rounds to the even state $|E\rangle_{a, b, c}$ state for even rounds. They can do this simply by performing Hadamard gates on their respective states at the end of every round of the protocol. The reason is relation (11). Thus they start the first round with the GHZ state $|G\rangle$ and end up with the $|E\rangle$ state which is used for the second round. At the end of the second round they have produced again the GHZ state $|G\rangle$ which will be used for the next round and so on.

We now assume that Eve who may be a collaborator of one of the receivers say Bob follows a more complicated strategy by entangling her system (ancilla) with the states of Alice, Bob and Charlie in the most general form, that is:

$$
|\Theta\rangle_{a, b, c, e}=\sum_{i, j, k}|i, j, k\rangle_{a b c} \otimes \eta_{i j k} \quad i, j, k=0,1 .
$$

where $\eta_{i, j, k}$ 's are un-normalized states of Eve. Eve wants to make this entanglement so that at the end of each round of sending and receiving a bit, useful information about that bit is collected in her ancilla which she can measure safely later on. Consider an odd round of the process and suppose that the starting state of Alice, Bob and Charlie, ignorant of the presence of Eve is as above. Eve is clever enough to entangle her state such that she does not perturb the values of the final bits measured by Bob and Charlie when the protocol is run for this round. (otherwise a comparison of a substring of bits between Alice and Bob and Charlie, will reveal her presence or her collaboration with one of the receivers.). She then finds that the ideal form of 
entanglement is as follows:

$$
\left|\Theta^{\text {odd }}\right\rangle=|0,0,0\rangle \eta_{000}+|1,1,1\rangle \eta_{111}
$$

where we have suppressed the subscripts on the states and the $\otimes$ symbol. If she keeps any other state in (10), say a state like $|0,1,1\rangle \eta_{011}$, the total superposition will have a term $|0,1,1, q, q\rangle \eta_{011}$ which reveals the encoded bit $q$ by Alice as $1+q$ to Bob and Charlie. Or if she keeps a state like $|0,1,0\rangle \eta_{010}$, the total superposition will have a term $|0,1,0, q, q\rangle \eta_{011}$ which reveals the encoded bit $q$ by Alice as $1+q$ to Bob and as $q$ to Charlie. In all cases these lead to her detection after a subsequence of bits are compared. A similar analysis reveals to Eve that the ideal form of entanglement for an even round of the protocol is:

$$
\left|\Theta^{\text {even }}\right\rangle=|0,0,0\rangle \xi_{000}+|1,1,0\rangle \xi_{110}+|1,0,1\rangle \xi_{101}+|0,1,1\rangle \xi_{011}
$$

The crucial point is that the Hadamard gates at the end of each round do not allow Eve to have desirable entanglement for every round. Eve can have desirable entanglement only if $H^{\otimes 3}\left|\Theta^{\text {even }}\right\rangle=\left|\Theta^{\text {odd }}\right\rangle$ and vise versa. A simple calculation yields:

$$
\begin{aligned}
H^{\otimes 3}\left|\Theta^{o d d}\right\rangle & =\frac{1}{2 \sqrt{2}}(|0,0,0\rangle+|110\rangle+|1,0,1\rangle+|011\rangle)\left(\eta_{000}+\eta_{111}\right) \\
& +\frac{1}{2 \sqrt{2}}(|1,1,1\rangle+|0,0,1\rangle+|010\rangle+|100\rangle)\left(\eta_{000}-\eta_{111}\right) .
\end{aligned}
$$

Equating this to $\left|\Theta^{\text {even }}\right\rangle$ yeilds $\eta_{000}=\eta_{111}$ and $\xi_{000}=\xi_{110}=\xi_{101}=\xi_{011}$. Looking back at (10) and (11), we see that this implies that this switching between desirable entanglement at alternative rounds is possible for Eve only if there is no entanglement at all in any of the rounds! One may argue that Eve may not want to completely avoid any error introduced into the data and she may entangle her system to the carriers in order to reduce the quantum bit error rate (QBER) as low as possible, lower than the expected level of noise and hence escape the detection. We will show in the appendix, that Eve can not lower the QBER averaged over odd and even rounds, below 25 percent. In this way we have shown the security of the protocol against Eve's attack or Bob's Cheating.

In conclusion we have presented a new protocol for quantum secret sharing based on reusable entangled states. In our protocol a sequence of bits is transmitted to two parties so that they can recover half of the bits independently and for the rest half they need to collaborate to find the identity of the bits. The distinctive feature of this protocol is the existence of a carrier which carries the data bits from the sender to the receiver in secure form, without any need for measurements in random bases and public announcements. We have also discussed the security of the protocol against evesdropping and against cheating of one of the receivers, and have shown that any such action leads to high error rates in the sequence of bits between the participants. A rigorous proof of the security of the protocol is however beyond the scope of the present work. 


\section{Appendix}

This appendix is a completion of section 3, where we show that even if we Eve allows a small quantum bit error rate introduced into the data, she can not find any form of entanglement of her system to the carriers to achieve this goal. Suppose that in the odd and even rounds Eve entangles her system to the carriers in the following general forms:

$$
\left|\Theta^{\text {odd }}\right\rangle=|0\rangle \eta_{0}+|1\rangle \eta_{1}+|2\rangle \eta_{2}+|3\rangle \eta_{3}+|4\rangle \eta_{4}+|5\rangle \eta_{5}+|6\rangle \eta_{6}+|7\rangle \eta_{7}
$$

and

$$
\left|\Theta^{\text {even }}\right\rangle=|0\rangle \xi_{0}+|1\rangle \xi_{1}+|2\rangle \xi_{2}+|3\rangle \xi_{3}+|4\rangle \xi_{4}+|5\rangle \xi_{5}+|6\rangle \xi_{6}+|7\rangle \xi_{7} .
$$

where for simplicity we have used binary notation and suppressed indices, i.e. $|0\rangle \xi_{0} \equiv$ $|000\rangle_{a b c} \otimes \xi_{0},|3\rangle \xi_{3} \equiv|011\rangle_{a b c} \otimes \xi_{3}$ etc. In order to reduce the QBER (the probability of unwanted bits introduced into the transferred bits) below a tolerable threshold $\epsilon$, she should choose the states such that:

$$
\left|\eta_{1}\right|^{2}+\left|\eta_{2}\right|^{2}+\left|\eta_{3}\right|^{2}+\left|\eta_{4}\right|^{2}+\left|\eta_{5}\right|^{2}+\left|\eta_{6}\right|^{2} \leq \epsilon, \quad \text { or } \quad\left|\eta_{0}\right|^{2}+\left|\eta_{7}\right|^{2} \geq 1-\epsilon
$$

in the odd rounds and

$$
\left|\xi_{1}\right|^{2}+\left|\xi_{2}\right|^{2}+\left|\xi_{4}\right|^{2}+\left|\xi_{7}\right|^{2} \leq \epsilon
$$

However the action of Hadamard gates relates the two states $\left|\Theta^{\text {odd }}\right\rangle$ and $\left|\Theta^{\text {even }}\right\rangle$ and hence the states $\left\{\eta_{i}\right\}$ and $\left\{\xi_{i}\right\}$. It is easy to find that under the Hadamard operations:

$$
\begin{aligned}
\xi_{1} & =\frac{1}{2 \sqrt{2}}\left(\eta_{0}+\eta_{1}-\eta_{2}-\eta_{3}-\eta_{4}-\eta_{5}+\eta_{6}+\eta_{7}\right) \\
\xi_{2} & =\frac{1}{2 \sqrt{2}}\left(\eta_{0}-\eta_{1}+\eta_{2}-\eta_{3}-\eta_{4}+\eta_{5}-\eta_{6}+\eta_{7}\right) \\
\xi_{4} & =\frac{1}{2 \sqrt{2}}\left(\eta_{0}+\eta_{1}+\eta_{2}+\eta_{3}-\eta_{4}-\eta_{5}-\eta_{6}-\eta_{7}\right) \\
\xi_{7} & =\frac{1}{2 \sqrt{2}}\left(\eta_{0}-\eta_{1}-\eta_{2}+\eta_{3}-\eta_{4}+\eta_{5}+\eta_{6}-\eta_{7}\right)
\end{aligned}
$$

A simple rearrangement yields

$$
\begin{aligned}
& \left|\xi_{1}\right|^{2}+\left|\xi_{2}\right|^{2}+\left|\xi_{4}\right|^{2}+\left|\xi_{7}\right|^{2}= \\
& \frac{1}{2}\left(\left|\eta_{0}-\eta_{4}\right|^{2}+\left|\eta_{1}-\eta_{5}\right|^{2}+\left|\eta_{2}-\eta_{6}\right|^{2}+\left|\eta_{3}-\eta_{7}\right|^{2}\right) \\
& \geq \frac{1}{2}\left(\left|\eta_{0}-\eta_{4}\right|^{2}+\left|\eta_{3}-\eta_{7}\right|^{2}\right) \simeq \frac{1-\epsilon}{2}
\end{aligned}
$$

where in the last line we have used (15). Therefore we see that keeping the QBER below a very small threshold $\epsilon$ in the odd rounds, will introduce a QBER of about 50 percent in the even rounds and vice versa. The average QBER over the odd and even rounds is $\frac{1}{2}\left(\epsilon+\frac{1-\epsilon}{2}\right)=\frac{1+\epsilon}{4}$ and the best that Eve can do is to minimize this average to 25 percent by minimizing $\epsilon$. 


\section{References}

1. I. V. Volovich and Ya. I. Volovich, On classical and quantum cryptography, quant-ph/0108133.

2. N. Gisin, G. Ribordy, W. Tittel and H. Zbinden; Quantum Cryptography, Rev. Mod. Phys. 74, 145 (2002).

3. M. Hillery, V. Buzek and A. Berthiaume; Phys. Rev. A 59, 1829 (1999).

4. C. H. Bennet and G. Brassard, in Proceeding of the IEEE International Conference on Computers, Systems, and Signal Processing, Banglore, India, 1984 (IEEE, New York, 1984), p. 175.

5. A. K. Ekert; Phys. Rev. Lett.,67, 661(1991).

6. D. M. Greenberger, M. A. Horne, and A. Zeilinger; Bell's theorem, Quantum Theory and The Conceptions of The Universe (M. Kafatos, ed. Kluwer Academic, Dor-drecht, the Netherlands, 1989).

7. Y. S. Zhang, C. F. Li, and G. C. Guo; Phys. Rev. A, 64, 024302 (2001).

8. A. Karlsson, M. Koashi, and N. Imoto; Phys. Rev. A 59, No. 1, 162 (1999).

9. D. Leibfried et al. Experiments towards quantum information with trapped Calcium ions; quant-ph/0009105

10. C. Cabrillo, J. I. Cirac, P. Garca-Fernndez, and P. Zoller Phys. Rev. A 59, 1025 (1999). 